\title{
Viable System Verilog Assertions(SVA) Praxis in AMBA AHB-Lite Protocol Design
}

\author{
Seerapu Anil Nagendra, Pallavi, M. Murali Krishna
}

\begin{abstract}
Digital integrated circuit(ic design entanglement has been far reaching since the demonstration by kil by in 1958.in this day and age system on chip(soc) design verification accommodate billion, more specifically trillion transistors designs came into existence due to artificial intelligence(ai) designs. The expertise designers tried to ramp up design process by using effective eda tools, still the time wheel move in recursive process. In order to accelerate time wheel of design process specified design methodology needed for every design. The overview of various design methodologies followed in the market now a days. Emulation performance by using veloce platform in bfm mode on ahb lite transmissions. Simulation by using software eda tools is slow going on wheel of design when we go for higher abstraction. Accelerated simulation and emulation using hardware is costly in contrast with software simulation. Prototyping is expedient. Formal verification and intelligent software simulations are frail. The possibility of selection between various hardware engines become ravelled. It develop into perspicuous only amalgamation of engines will assist design verification teams to be triumphant. In this design combination of hardware accelerated simulator as a combination of emulator used to accelerate time wheel by using arm amba ahb lite protocol as a design.
\end{abstract}

Keywords: AMBA, AHB LITE, Simulation, Emulation, Assertions

\section{INTRODUCTION}

In this day and age many new vendors begin to develop their own digital IC intellectual property. one of those is on ARM and each vendor come up with the needed solution to integrate their electronic devices. these Intellectual property evolve with the help of electronic design automation (EDA)tools in order to pull off the design performance and other tradeoffs such as area , p9wer , and design time . the EDA tools routed by scripting algorithms developed by the design engineers and they self executing many parts of the design verification

Revised Manuscript Received on July 05, 2020.

* Correspondence Author

S.Anil Nagendra*, VLSI Design(M Tech), GITAM University, Visakhapatnam, India.

Pallavi, VLSI And Embedded System (M Tech), RNSIT, BANGLORE, India. E-mail: Sugandhi2391@Gmail.Com

M. Murali Krishna, ECE Department, GITAM University, Visakhapatnam, India.

(C) The Authors. Published by Blue Eyes Intelligence Engineering and Sciences Publication (BEIESP). This is an open access article under the CC BY-NC-ND license (http://creativecommons.org/licenses/by-nc-nd/4.0/) flow. so the design verification teams are more with hands on experience make design verification time wheel less and time to market is fast. the arm ahb lite protocol taken from arm site modified according to the design requirements and make sure that after modified design works properly and it had the same operation states that is integrated in ARM IP's. Detailed evaluation of design verification methodologies in market and comparison of methodologies, simulation tools of various vendors. Emulation platform working nature on SoC design SVA(System Verilog Assertions) usage in UVM Environment DUT

\section{AHB LITE PROTOCOL}

AHB Lite system can be single master multiple slave as shown in fig.1. Or multi layer AHB system in which only one ahb master is used.AHB Lite is extension of ahb protocol and deliberately useful in system where only need a single bus master.

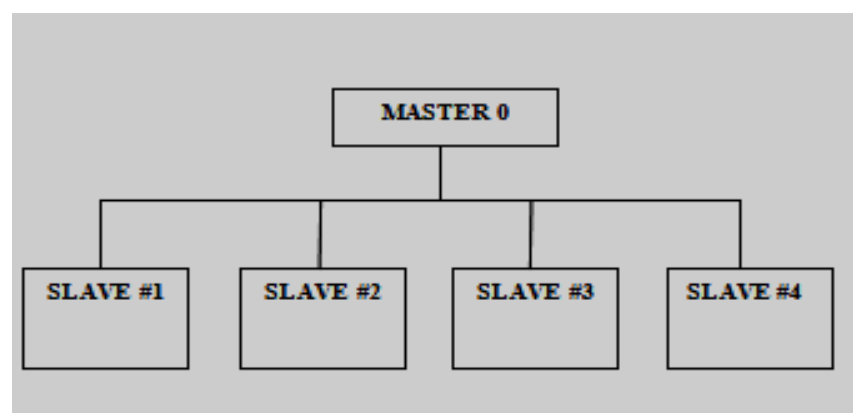

Fig.1.AHB Lite system

Fig.2. shows AHB Lite protocol architecture which consists of Single master module and three slave modules inn conjunction with the bus interconnection by using decoder and multiplexer.

operation:

when read or write transfer initiated by either master or slave by using address, control signals. those are communicate with decoder to obtain address and direction, size of the transfer . Transfers can be incrementing , wrapping , undefined length , burst transfers

the transaction consists of two operation phases which are Address transaction phase and Data transaction phase the write data bus operation initiated by master and written up in slave. read data bus initiated by slave and reads data from master.

HREADY signal used to develop wait states and slave has more time to sample data .

Published By:

Blue Eyes Intelligence Engineering DOI: 10.35940/ijeat.E1096.089620

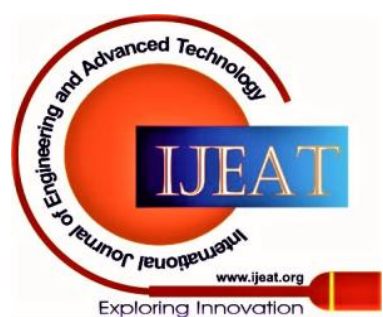





Slave

Fig.2.AHB LITE PROTOCOL ARCHITECTURE

Fig.3. shows AHB Lite slave Top Module

AHB Lite slave uses HSELx signal generated by decoded to response the bus transactions controlled master in the protocol. Slave proffer Response either Success ,failure, or waiting transfers

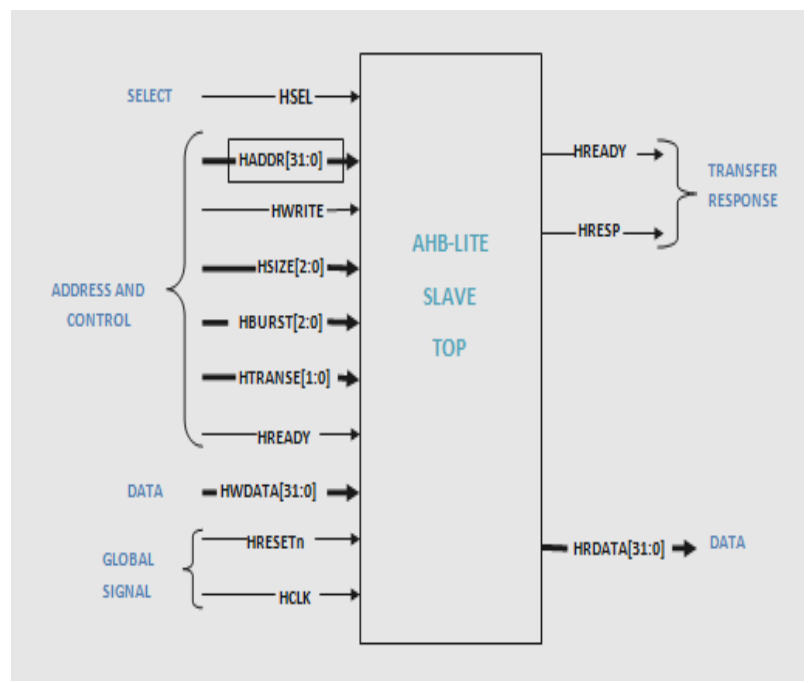

Fig.3.AHB LITE SLAVE TOP

\section{ENVIRONMENT}

Master: is written/programmed using a Bus Functional Model Approach (not Synthesizable)

-It contains a host of tasks -read, write-that encompass the way the DUT is used.

-By calling those tasks a read or a write would occur on the bus/interface of the DUT

Slave: number of memory modules used in design is parameterized. slave is a RAM, simple memory module.

Monitor: The Current Transactions developed by assertions monitored by using this module and also checks the bus traffic signals.

Interface: Slave MODPORT provide a proper lookup for the slave.
Fig.4.shows SV design verification environment used in simulation and emulation and all the AHB Lite bus signals described in it

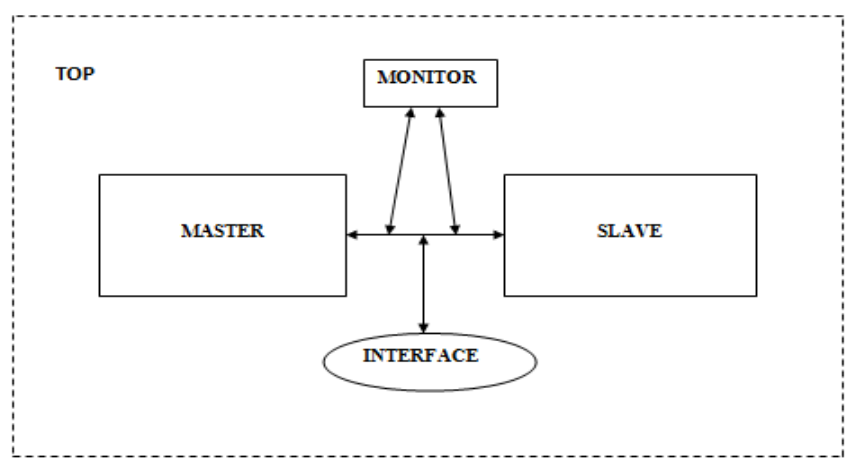

Fig.4.SYSTEM VERILOG ENVIRONMENT

\section{VERIFICATION PLAN AND TEST CASES}

property error_check;

@(posedge Bus.HCLK) disable iff (Bus.HTRANS == BUSY

$\|$ Bus.HTRANS == IDLE)

(Bus.HADDR > ((2**10)* `NoOfSlaves))|-> (Bus.HRESP

$==1 ' \mathrm{~b} 1$ );

endproperty

property read_only_error_check;

@(posedge Bus.HCLK) disable iff ( (Bus.HADDR[9:0] > 9'd3))

(Bus.HWRITE ==1'b1) $\mid=>($ Bus.HRESP ==1);

endproperty

property basic_write;

@(posedge Bus.HCLK) disable iff ((Bus.HTRANS==IDLE ||

Bus.HTRANS==BUSY) \&\& Bus.HBURST>'0)

\$rose(Bus.HWRITE) |=> Bus.HREADY;

endproperty.

TEST CASES

InterfaceInstance.burst_write (32'd5,4,0, data_burst); \#20;

InterfaceInstance.burst_read (32'd5,4,0,

data_burst_read);

\#20;

ifdef DEBUG

\$display ("DATA - 0 BUSY - BURST4 = \%d, \%d, \%d, $\% d \backslash n "$, data_burst_read[0], data_burst_read[1],

data_burst_read[2], data_burst_read[3] ); `endif

InterfaceInstance.burst_write (32'd10,8,0, data_burst); \#20;

InterfaceInstance.burst_read (32'd10,8,0,

data_burst_read);

\#20;

ifdef DEBUG

\$display ("DATA - 0 BUSY - BURST8 = \%d, \%d, \%d, $\% \mathrm{~d}, \% \mathrm{~d}, \% \mathrm{~d}, \% \mathrm{~d}, \% \mathrm{~d} "$, data_burst_read[0],

data_burst_read[1], data_burst_read[2], data_burst_read[3], data_burst_read[4], data_burst_read[5], data_burst_read[6], data_burst_read[7]);

`endif

InterfaceInstance.burst_write (32'd25,16,0, data_burst); \#20;

Published By:

Blue Eyes Intelligence Engineering

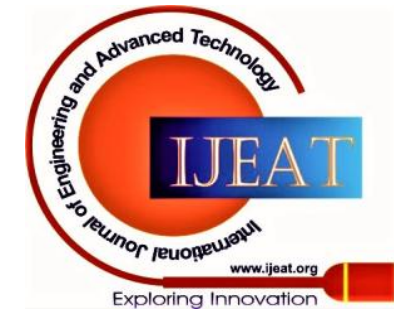


InterfaceInstance.burst_read (32'd25,16,0,

data_burst_read);

\#20;

iifdef DEBUG

\$display ("DATA - 0 BUSY - BURST16 = \%d, \%d, \%d, \%d, \%d, \%d, \%d, \%d, \%d, \%d, \%d, \%d, \%d, \%d, \%d, \%d", data_burst_read[0], data_burst_read[1], data_burst_read[2], data_burst_read[3], data_burst_read[4], data_burst_read[5], data_burst_read[6], data_burst_read[7], data_burst_read[8], data_burst_read[9], data_burst_read[10], data_burst_read[11], data_burst_read[12], data_burst_read[13], data_burst_read[14], data_burst_read[15]); ‘endif

\section{RESULTS}

\section{ASEERTION SCOREBOARD WITHOUT ERRORS}

\begin{tabular}{|c|c|c|c|}
\hline \multicolumn{4}{|c|}{  } \\
\hline TYPE OF CISCX & TOEAL CONGT & Pass COARI & an coosr \\
\hline error_check & 10 & 10 & 0 \\
\hline read onlp_error_chects & 6 & 6 & 0 \\
\hline basic_vrite_error & 34 & 34 & 0 \\
\hline bagic__read_error_check & 20 & 20 & 0 \\
\hline basic_burst_uritē_check & 32 & 32 & 0 \\
\hline basic_burst_raad_check & 35 & 35 & 0 \\
\hline ARENUI_becil - & 12 & 12 & 0 \\
\hline busy_to sequential_check & 35 & 35 & 0 \\
\hline Sequential_vait_cheeck & 11 & 11 & 0 \\
\hline bursts_coint_cheeckd & 10 & 10 & 0 \\
\hline bursts coumt checks & 10 & 10 & 0 \\
\hline bursts_coumt_checllo & 12 & 12 & 0 \\
\hline address_cthanget & 13 & 13 & 0 \\
\hline adoress_changee & 25 & 25 & 0 \\
\hline address__hangel16 & 24 & 24 & 0 \\
\hline
\end{tabular}

\section{ASEERTION SCOREBOARD WITH ERRORS}

\begin{tabular}{|c|c|c|c|}
\hline \multicolumn{4}{|c|}{ | } \\
\hline TYPE OF CIIICK & TOLAL COUNT & PASS CXOMTI & FAIL COUUT \\
\hline f error checl & 96 & 15 & 81 \\
\hline | read only error check & 11 & 9 & 2 \\
\hline I basic_vrite_error & 23 & 23 & 0 \\
\hline \# basic_read_error_check & 20 & 20 & 0 \\
\hline f basic_burst_ vritēecheck & 21 & 21 & a \\
\hline I basic_burst_read_theck & 36 & 36 & 0 \\
\hline 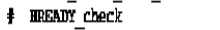 & 12 & 12 & 0 \\
\hline \& busy_to_sequential_check & 19 & 19 & $a$ \\
\hline I Sequential_kait_check & 122 & 111 & 11 \\
\hline f bursts count checkt & 10 & 10 & 0 \\
\hline f bursts count checks & 10 & 10 & 0 \\
\hline \# bursts_court_check16 & 12 & 12 & 0 \\
\hline f address_change4 & 13 & 13 & 0 \\
\hline f address change8 & 25 & 25 & a \\
\hline If address_changel6 & 24 & 24 & 0 \\
\hline
\end{tabular}

\section{Emulation -veloce}

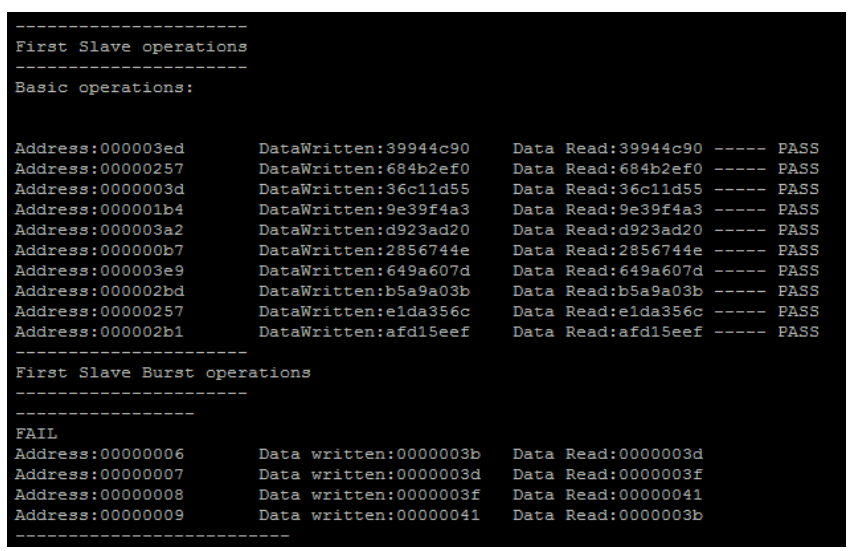

Published By:

Blue Eyes Intelligence Engineering and Sciences Publication

(C) Copyright: All rights reserved.

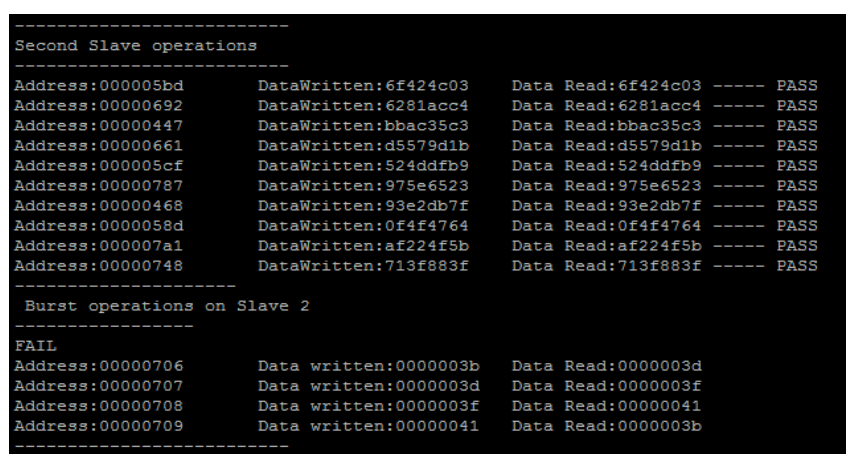

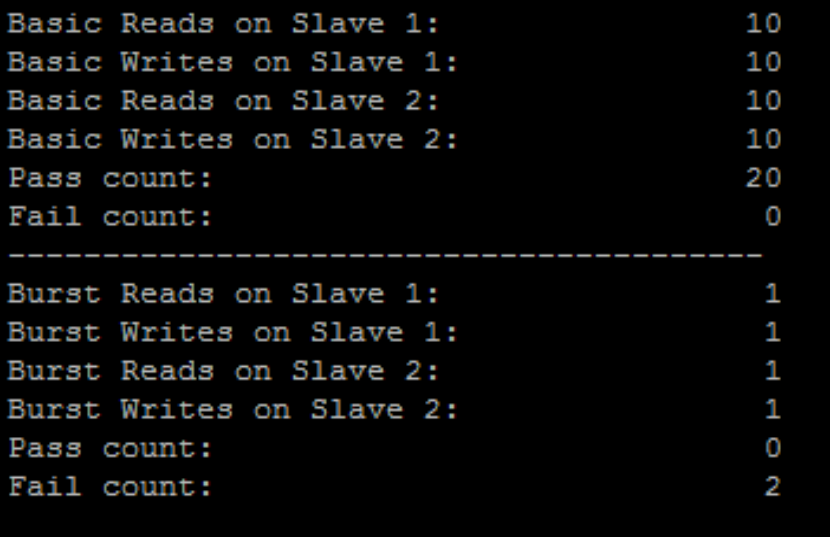

\section{CONCLUSIONS}

the initial functionality defining RTL code written from AHB lite specifications taken from ARM documentation. which is exhaustively created test bench environment simulated by using Questasim tool.

design verification issues rapidly increases day by day due to radically increasing in gate count and functionalities . more than $75 \%$ time , resources and endeavor takes in detecting bugs in interconnection on SoC chipsets is boundless problem.

simulation and emulation engines combination is unique and SV methodologies proffer accelerated performance ,flexible design, scalability in design area. these methods support real test environment for the design under test (DUT).By wrapping the emulation DUT platform via intelligent interfaces with components enables high speed in design verification.

the systems had high level abstractions can be debugged easily by co emulation constructed test bench. the design verification teams moves to strategic development to accelerate the design performance and time to market

An acceleration ready frame work describes benefits in yielding higher gain over pure simulation and significant decrease in development time for emulation. Users will be able to write block level SV environment that can be uses directly in emulation. this approach will be a solution in getting SoC design verification in shorter times for block, subsystem, system level. 


\section{Viable System Verilog Assertions(SVA) Praxis in AMBA AHB-Lite Protocol Design}

\section{FUTURE SCOPE}

in the day and age SoC designs are high level abstraction based with innumerable functionalities. to verify SoC deign the teams required more and correlation between each team is mandatory.

If we take a simulator as the baseline, emulation, acceleration and prototyping cam all run faster than a simulator, but the speed-up is dependent on many factors AI SoC Development engineers grasp the design verification environment which will reduce the design services time wheel to come into market .while generating these new generation IP's and soc chipsets .which are highly built in advantages over currently used IP's and soc chipsets

design verification grasp by AI technology makes accelerate the design time and availability in market , which will provide extra time to design teams to make clear cut design features.

\section{REFERENCES}

1. AHB, AMBA Multi-Layer. "AHB-Lite,"." Bus Specification http://www. arm. com.

2. Taraate, Vaibbhav. "Buses and Protocols in SOC Designs." In Advanced HDL Synthesis and SOC Prototyping, pp. 97-117. Springer, Singapore, 2019.

3. Mulani, Purvi D. "SoC level verification using System Verilog." In 2009 Second International Conference on Emerging Trends in Engineering \& Technology, pp. 378-380. IEEE, 2009.

4. Foster, Harry, Lawrence Loh, Bahman Rabii, and Vigyan Singhal. "Guidelines for creating a formal verification testplan." Proc. DVCon (2006).

5. Cohen, Ben, Srinivasan Venkataramanan, and Ajeetha Kumari. SystemVerilog Assertions Handbook:--for Formal and Dynamic Verification. vhdlcohen publishing, 2005.

6. Shah, Saurin, and Nirav Patel. "Systemverilog Based AMBA AHB Verification Environment." International Journal of Engineering Research 3, no. 5 (2014).

7. Gandhi, Ashima. "Functional Verification of AMBA AHB-Lite using Layered Testbench Technology of System Verilog." Journal of VLSI Design Tools \& Technology 6, no. 2 (2016): 104-112.

8. Kante, Sravya, Hari Kishore Kakarla, and Avinash Yadlapati. "Design and Verification of AMBA AHB-Lite protocol using Verilog HDL." International Journal of Engineering and Technology, EISSN 0975-4024: 734-741.

9. Sinha, Richa, Akhilesh Kumar, and Archana Kumari Sinha. "Verification analysis of AHB-LITE protocol with coverage." International journal of advances in Engineering \& technology 2, no. 1 (2012): 121.

10. M. B. R. Srinivas and S. Musala, "Verification of AHB_LITE protocol for waited transfer responses using re-usable verification methodology," 2016 International Conference on Inventive Computation Technologies (ICICT), Coimbatore, 2016, pp. 1-3, doi: 10.1109/INVENTIVE.2016.7830137.

11. A. K. Singh, A. Shrivastava and G. S. Tomar, "Design and Implementation of High Performance AHB Reconfigurable Arbiter for Onchip Bus Architecture," 2011 International Conference on Communication Systems and Network Technologies, Katra, Jammu, 2011, pp. 455-459, doi: 10.1109/CSNT.2011.99.

12. Goswami, Disha, and Dharmesh J. Shah. "Implementation of SystemVerilog Environment for Functional Verification of AHB-DMA Bridge." International Journal on Recent and Innovation Trends in Computing and Communication 3, no. 5: 2799-2802.

\section{AUTHORS PROFILE}

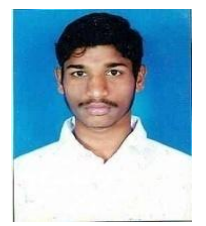

Seerapu Anil Nagendra Pursuing $M$ Tech in VLSI Design from GITAM Deemed to be University, Visakhapatnam. Currently Doing Internship as SoC DV , SION SEMICONDUCTORS PVT LTD, Bangalore.

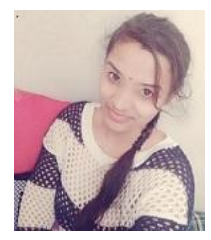

Pallavi Completed B.Tech In ECE From Godutai Engineering College For Women, Kalaburigi. Currently Pursuing M.tech in VLSI and Embedded systems from RNSIT, Bangalore.

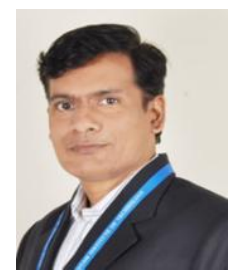

Muralikrishna. M working as Asst. professor in Dept of ECE,GIT,GITAM deemed to be university. Had hands on experience in various VLSI Design methodologies and Worked on Analog IC design as well as Digital IC design methodology flows. 